\title{
A study of reaction $K^{-} N \rightarrow\left(K^{-} \pi^{+} \pi^{-}\right) N$ at $28 \mathrm{GeV} / \mathrm{c}$
}

\author{
V.Nikolaenko ${ }^{*}$ for VES experiment ${ }^{\dagger}$ \\ Address \\ Institute for High Energy Physics, 142281 Protvino, Moscow reg., Russia \\ E-mail: nikolaenko@mx. ihep.su !
}

Abstract: Diffractive-like production of strange meson resonances is studied at the statistics of $\sim 300000$ events, which exceeds statistics in previous experiments. Experimental data have been acquired by VES spectrometer, exposed in unseparated tagged beam off Serpukhov accelerator on Be target. Preliminary results of the standard 3meson Partial Wave Analysis (PWA) of $\left(K^{-} \pi^{+} \pi^{-}\right)$system with mass below $2 \mathrm{GeV}$ are presented. Apart from two established resonances with spin-parity $J^{P}=1^{+}, K_{1}(1270)$ and $K_{1}(1400)$, we have indications on the 3 -rd resonance with mass close to $1750 \mathrm{MeV}$. Also properties of pseudoscalar resonance, $K_{0}(1460)$ are discussed.

The properties of $\left(K^{-} \pi^{+} \pi^{-}\right)$-system have been studied in several experiments, two of them are the most statistically significant: $K^{ \pm} p$ at $13 \mathrm{GeV} / \mathrm{c}$ [i] $(\sim 138000$ events in total) and $K^{-} p$ at $63 \mathrm{GeV} / \mathrm{c}$ [2] $(\sim 191000$ events). The total mass distribution has two wide bumps, near 1300 and $1800 \mathrm{MeV}$, called Q- and L-regions, respectively.

The wave with spin-parity $J^{P}=1^{+}$is dominant, particularly in the Q-region. There are two well known resonances here, $K_{1}(1270)$ and $K_{1}(1400)$, which are interpreted as a mixture of two $(s \bar{u})$-states with parallel and anti-parallel spins of quarks. Looking for the number of events in another waves, the second and the 3 -rd waves are the $J^{P}=0^{-}$and $2^{-}$ waves. A wide resonance with mass $\sim 1400-1460 \mathrm{MeV}$ has been observed in $0^{-}$wave, which is not yet considered as well established. Events in $2^{-}$wave are concentrated mainly in the L-region. It would be natural to expect two $2^{-}$mesons, like two $1^{+}$resonances in Q-region. There are published fit results for $2^{-}$wave with one or two resonances in the L-region in the $\left(K^{-} \pi^{+} \pi^{-}\right)$-system [i2], as well as for the $(K \omega)$-system [i- The $\chi^{2}$ probabilities are better for two resonance hypothesis, however the difference is not large and this question needs clarification.

Only $1^{+}$and $0^{-}$waves are discussed in this talk, according to the present status of the analysis.

${ }^{*}$ Speaker.

${ }^{\dagger}$ supported by grants INTAS-RFBR 97-02-71017, RFBR 00-02-16555 and VNS-RFBR 00-15-96689. 
VES facility is a wide angle magnetic spectrometer designed for studies of light meson resonances. It is installed in a beam of unseparated negative particles with possible momentum range between 23 and $46 \mathrm{GeV} / \mathrm{c}$ off the IHEP accelerator. Reconstruction of charged tracks is provided by a system of proportional and drift chambers. The spectrometer is equipped with a lead-glass electromagnetic calorimeter and with a gas Cherenkov detector, which is suitable for identification of secondary $K^{ \pm}$in the momentum range from $\sim 4.5$ to $\sim 16 \mathrm{GeV} / \mathrm{c}$. A system of three beam Cherenkov detectors provides the identification of initial $\pi^{-}, K^{-}$and $\bar{p}$. A minimum bias trigger is used, which is based on a system of scintillator hodoscopes.

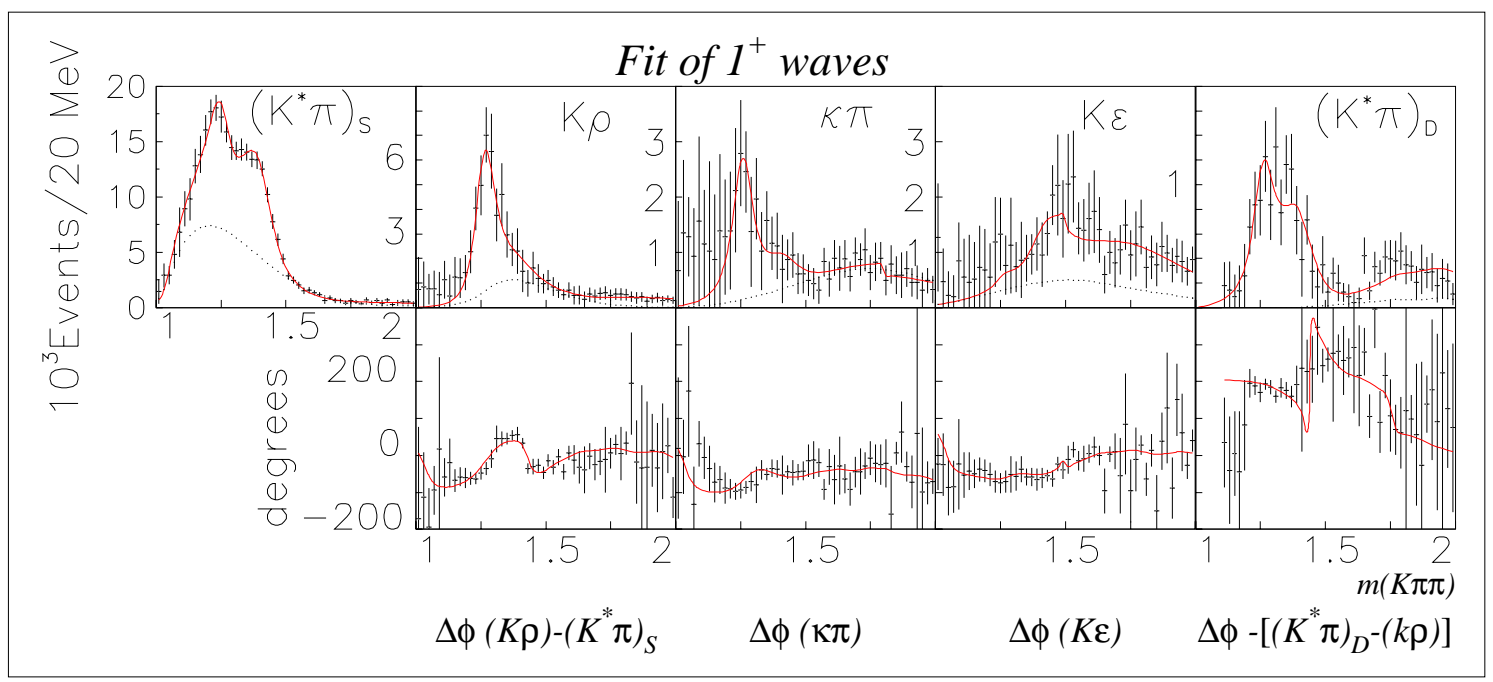

Figure 1: PWA results for $J^{P} M^{\eta}=1^{+} 0^{+}$waves, $1.0<m(K \pi \pi)<2.0 \mathrm{GeV}, 0<|t|<0.7 \mathrm{GeV}$. Number of events in $20 \mathrm{MeV}$ bins and relative phases are shown. Dotted lines show contributions from Deck effect. Phases are measured with respect to $\left(K^{*} \pi\right)_{S}$, excepting the $\left(K^{*} \pi\right)_{D}$ channel.

The following selection criteria have been applied:

- kaon beam (no signals in two beam Cherenkov detectors tuned below K-threshold; but a signal seen in the 3-rd one, above K-threshold);

- there are 3 charged secondary tracks (one positive, two negative);

- the energy sum of charged tracks is in the range $(25 ., 30$.) $\mathrm{GeV}$;

- there is no $\pi^{0}, \eta$ in the event and the total energy deposited in the Electromagnetic Calorimeter is consistent with noise;

- the momentum transfer cut: $\left|t^{\prime}\right|<0.7 \mathrm{GeV}^{2}$;

- at least one secondary negative track is identified by Cherenkov detector of secondary tracks as $K^{-}$or $\pi^{-}$.

- rejected events with two identified $\pi^{-}$or two $K^{-}$or with identified $K^{+}$. 


\begin{tabular}{|c|c|c|c|}
\hline & $K_{1}(1270)$ & $K_{1}(1410)$ & $K_{1}(1750)$ \\
\hline mass, $M e V$ & $1274 \pm 9$ & $1407 \pm 10$ & $1755 \pm 50$ \\
\hline width, $M e V$ & $45 \pm 8$ & $83 \pm 10$ & $269 \pm 90$ \\
\hline$B r\left(K^{*} \pi\right)_{S}, \%$ & $16.1 \pm 4.1$ & $80.6 \pm 9.1$ & $9.7 \pm 1.0$ \\
\hline$B r(K \rho), \%$ & $65.7 \pm 7.3$ & $6.9 \pm 5.7$ & $26.5 \pm 11.1$ \\
\hline$B r(\kappa \pi), \%$ & $9.2 \pm 0.9$ & $1.4 \pm 0.2$ & $4.5 \pm 1.8$ \\
\hline$B r(\epsilon K), \%$ & $1.1 \pm 0.4$ & $3.6 \pm 1.0$ & $32.2 \pm 6.8$ \\
\hline$B r\left(K^{*} \pi\right)_{D}, \%$ & $7.8 \pm 1.2$ & $7.8 \pm 1.9$ & $27.0 \pm 3.9$ \\
\hline
\end{tabular}

Table 1: Preliminary parameters of resonances in $1^{+}$wave.

- rejected events if $\pi^{ \pm}$or K-meson has $\cos \theta_{G J}<-0.92$ in the Gotfried-Jackson ref. frame, in order to exclude events with $(p \pi)$ or $\left(p K^{-}\right)$isobars.

Selected sample contains 300895 events with masses of $\left(K^{-} \pi^{+} \pi^{-}\right)$system in the range $(1.0,2.1) \mathrm{GeV}$. An analysis of the sample purity has shown that the most serious impurity comes from the $\left(\pi^{-} \pi^{+} \pi^{-}\right)$system produced in $K^{-}$beam, this admixture is of order of $5 \%$. Admixtures due to the beam misidentification and from the $\left(K^{-} K^{+} K^{-}\right)$channel are negligible.

At the next step, the 3-meson PWA program was used (the program which has been

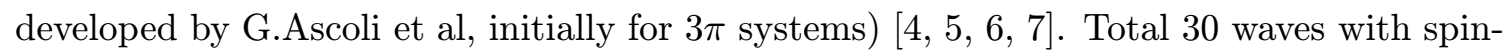
parity $1^{+}, 0^{-}, 2^{-}, 3^{+}, 4^{-}, 2^{+}$and $1^{-}$were included with intermediate isobars $K^{*}(890)$, $\rho, \epsilon$ (excluded $\left.f_{0}(980)\right), \kappa$ or $K_{0}(1430), K^{* *}(1430)$ or $K_{2}(1430), f_{2}(1270), K_{3}(1780)$, and $f_{0}(980)$. Like the ACCMOR experiment [2: 5 decay modes were included for $1^{+}$wave. Constructing the density matrix, all 5 channels for this wave were taken as connected with separate diagonal elements of the $\rho$-matrix, i.e. we have not assumed that some of $1^{+}$waves

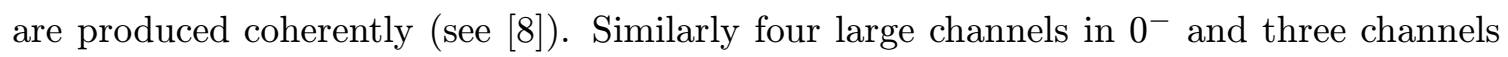
in $2^{-}$wave are taken without coherence assumption.

\begin{tabular}{|c|c|}
\hline & $K_{0}(1460)$ \\
\hline mass, $M e V$ & $1450 \pm 37$ \\
\hline width, $M e V$ & $110 \pm 25$ \\
\hline \hline$B r\left(K^{*} \pi\right), \%$ & $38.3 \pm 4.1$ \\
\hline$B r(K \rho), \%$ & $6.7 \pm 0.8$ \\
\hline$B r(\epsilon K), \%$ & $7.4 \pm 1.2$ \\
\hline$B r(\kappa \pi), \%$ & $47.7 \pm 5.1$ \\
\hline
\end{tabular}

Table 2: Preliminary parameters of resonance in $0^{-}$wave.

The PWA results for $1^{+}$channels are shown in Fig. 1. At the next step we fitted the mass spectra in 5 channels and Re/Im parts of non-diagonal elements of density matrix (or relative phases between channels). The model contains 3 resonances: $K_{1}(1270), K_{1}\left(1400, K_{1}(1750)\right.$ and the Deck effect amplitudes, all objects are taken as partially coherent. There is a complication with $K_{1}(1270$ resonance: the threshold in the dominant decay channel, $K \rho$. Therefore this resonance should have a deviation from the Breight-Wigner shape. This deviation was calculated following the method proposed in $\left[\bar{g}_{1}\right]$ for $a_{1}$ resonance. The analytically correct BW for decay $R \rightarrow a b$,

$$
B W_{c o r r}(s)=\frac{M \Gamma_{a b}(s)}{\left(s-m^{2}(s)\right)^{2}+\left(M \Gamma_{t o t}(s)\right)^{2}}
$$


A mass shift in the denominator of Breight-Wigner function arises:

$$
m^{2}(s)=M_{0}^{2}+\delta^{2}(s)
$$

the mass shift emerges from the real part of renormalisation term in the propagator,

$$
\delta^{2}(s)=\operatorname{Re} \Pi(s)=\frac{1}{\pi} \int_{s_{t h}}^{\infty} \frac{-\operatorname{Im} \Pi(s)}{s-s^{\prime}} d s^{\prime}
$$

here $s_{t h}=\left(m_{a}+m_{b}\right)^{2}$ and the $\operatorname{Im} \Pi(s)$ corresponds to the total width:

$$
\operatorname{Im} \Pi(s)=-M \Gamma_{t o t}(s)=-M \sum_{i} \Gamma_{i}(s) ;
$$

where $\Gamma_{i}(s)$ includes the phase space factor. At the case of decay in $S$-wave and into a pair of stable particles, the Cauchy integration can be performed explicitly, in elementary functions. The generalisation for the case of several decay channels, with different thresholds (or for the case with one of the decay products being a resonance) is evident.

We estimated the mass shift $\delta^{2}(s)$, starting from PDG values of the $K_{1}(1270)$ mass, width and branching ratios [i] $\left.{ }_{1}^{1} \overline{0}\right]$ for five measured channels and also $(K \omega)$ channel. Actually, the $\delta^{2}(s)$ term amplifies the difference between $K^{*} \pi$ and $K \rho$ decay channels, which exists already due to the phase space factors.

The Deck rescattering amplitudes were simulated by a method proposed in [i] ${ }_{1}^{1} \overline{1}_{1}^{1}$. Then the shapes of Deck amplitudes are kept, but the overall normalisation and the phase shift are considered as free parameters. Five mass spectra, $\operatorname{Re}\left(\rho_{i j}\right)$ and $\operatorname{Im}\left(\rho_{i j}\right)$ distributions are fitted. Satisfactory fit of all distribution, in the mass range from 1.0 to $2.0 \mathrm{GeV}$ is obtained, with $\chi^{2} / N D=217 . / 352$. Fit results are shown by solid lines in Fig. 1. Preliminary parameters of $1^{+}$resonances are given in Table 1 . The branching ratios are corrected for other decay modes, with $\pi^{0}$ or $K^{0}$. Errors in Table 1 include systematic errors from inclusion of small waves and different bining.

A similar procedure was applied for description of four channels in $0^{-}$wave. Here the model contains only one resonance, $K_{0}(1460)$. The phases of $0^{-}$waves were measured with respect to $1^{+}$waves. Fit results are presented in Fig. 2 and in Table $2\left(\chi^{2} / N D=130 . / 134\right)$. Like Table 1, the branching ratios here are corrected for other decay channels and errors include systematic uncertainties. There is no indication on the 2-nd resonance with a mass of 1800-1900 MeV, which could be expected as a strange partner of the $\pi(1800)$. One can notice a strong negative interference between resonance and Deck amplitude, particularly for $(\kappa \pi)$ channel. The difference in peak positions for $\left(K^{*} \pi\right)$ and $(K \rho)$ channels can be explained by interference with Deck amplitudes.

In conclusion, the main results of this study can be summarised as follows:

- Partial Wave Analysis of $\left(K^{-} \pi^{+} \pi^{-}\right)$system is performed on the statistics of $\sim 300000$ events. The general pattern of large waves is similar to the pattern observed in $K^{-} p$ experiment at $63 \mathrm{GeV} / \mathrm{c}$.

- A complex of 5 most significant $1^{+}$waves in the mass range from 1 to $2 \mathrm{GeV}$ can be described as a superposition of 3 resonances and Deck rescattering amplitudes. 


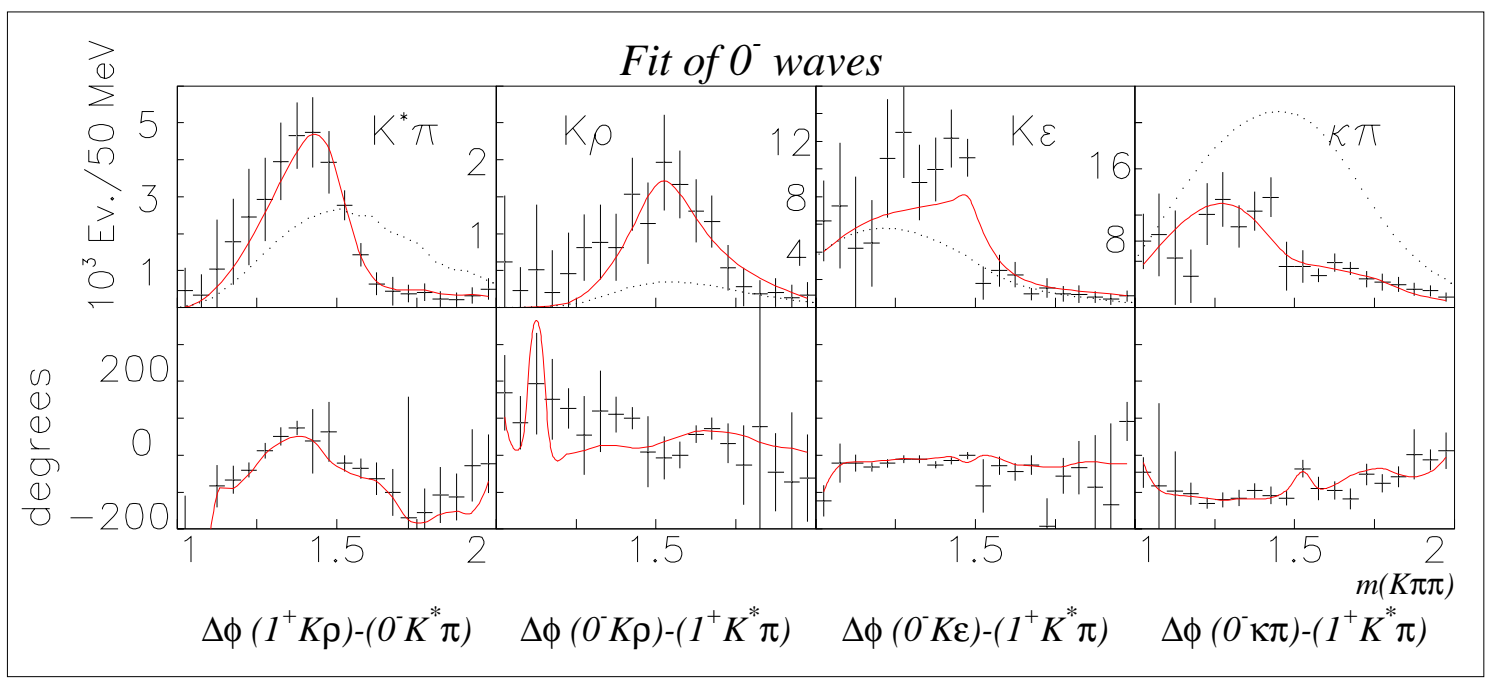

Figure 2: PWA results for $J^{P} M^{\eta}=0^{-} 0^{+}$waves, $1.0<m(K \pi \pi)<2.0 \mathrm{GeV}, 0<|t|<0.7 \mathrm{GeV}$. Number of events in $50 \mathrm{MeV}$ bins and relative phases are shown. Dotted lines show contributions from Deck effect.

Existence of $1^{+}$resonance with mass close to $1750 \mathrm{MeV}$ is confirmed. Preliminary values of mass, width and Branching ratios of this object are determined.

- A satisfactory description of 4 most significant channels of $0^{-}$wave below $m_{t o t}=2$ $\mathrm{GeV}$ requires one resonance, $K_{0}(1460)$ and Deck effect. Preliminary measurements of $K_{0}(1460)$ parameters are presented. There is no evidence for 2-nd resonance in this wave, a strange partner of $\pi(1800)$.

\section{References}

[1] G.W.Brandenburg et al, Phys. Rev. Lett. 36(1976) 703, 706; Nucl. Phys. B127(1977) 509

[2] C.Daum et al., Nucl. Phys. B187(1981)p.1, and ref. therein;

[3] D.Aston et al., Phys. Lett. B308(1993)p.186

[4] G.Ascoli et al., Phys. Rev. Lett. 25(1970)962;

[5] G.Ascoli et al., Phys. Rev. D7(1973)669;

[6] G.Ascoli et al., Phys. Rev. D9(1974)1963;

[7] D.V.Brockway, University of Illinois report COO-1195-197(1970);

[8] J.D.Hansen et al., Nucl. Phys. B81(1974)p.403;

[9] N.A.Tornqvist, Z.Phys.C36(1987) 695;

[10] Particle Data Group, Review of Particle Physics Eur. Phys. J C15(2000), p.530;

[11] G.Ascoli et al., Phys. Rev. D8(1973)3894;

[12] Particle Data Group, Review of Particle Physics Eur. Phys. J C15(2000), p.536-539; 